\title{
Preoperative cervical cytology as a prognostic factor in endometrioid-type endometrial cancer: a single-center experience from Saudi Arabia
}

\author{
Ahmed Abu-Zaid ${ }^{1,2}$, Mohannad Alsabban³, Osama Alomar ${ }^{1,3}$, Mohammed Abuzaid4, Mohammed Z. Jamjoom³, \\ Hany Salem ${ }^{1,3}$, Ismail A. Al-Badawi ${ }^{1,3}$ \\ ${ }^{1}$ College of Medicine, Alfaisal University, Riyadh, Saudi Arabia, ${ }^{2}$ College of Graduate Health Sciences, University of Tennessee Health Science \\ Center, Memphis, Tennessee, United States, ${ }^{3}$ Department of Obstetrics and Gynecology, King Faisal Specialist Hospital and Research Centre, \\ ${ }^{4}$ Department of Obstetrics and Gynecology, King Fahad Medical City, Riyadh, Saudi Arabia
}

\begin{tabular}{|c|}
\hline Access this article online \\
\hline Website: www.avicennajmed.com \\
\hline DOI: 10.4103/ajm.ajm_147_19 \\
\hline Quick Response Code: \\
\hline
\end{tabular}

\begin{abstract}
Objectives: The objectives of this study were (1) to estimate the frequency of preoperative abnormal cervical cytology (CC), (2) to explore correlations between preoperative CC and specific clinicopathological prognostic factors (tumor stage, endometrioid grade, myometrial invasion, lymphovascular space involvement, cervical involvement, and recurrence), and (3) to examine the impact of preoperative CC on disease-free survival (DFS) and overall survival (OS) in Saudi patients with endometrioid-type endometrial cancer (EC). Materials and Methods: A retrospective cross-sectional study was conducted at a tertiary hospital in Saudi Arabia. The study's inclusion criteria included: (1) patients who underwent staging operation for EC from 2010-2014, (2) patients who had preoperative CC results within 3 months before staging operation, and (3) patients with final histopathological diagnosis of endometrioid-type EC. Results: Hundred and sixteen patients $(n=116)$ met the study's inclusion criteria. CC results were abnormal in 46 patients (39.7\%). Patients with abnormal CC had statistically significant higher rates of unfavorable Grades II-III tumor and cervical involvement than patients with normal CC ( $P=0.004$, chi-square test). There were no statistically significant differences (logrank test) between patients with normal and abnormal CC with regard to DFS $(P=0.525)$ and OS $(P=0.166)$. Multivariate analyses of DFS and OS (Cox proportional hazards model) failed to show preoperative $\mathrm{CC}$ as a significant independent prognostic factor of DFS and OS $(P>0.05)$. Conclusion: The frequency of abnormal preoperative $\mathrm{CC}$ in patients with endometrioid-type $\mathrm{EC}$ is not uncommon. Abnormal CC correlates with poor prognostic factors, namely high tumor grade and cervical involvement. Preoperative CC is not a significant independent prognostic factor of survival.
\end{abstract}

Key words: Cervical cytology, endometrial cancer, pap smear, prognosis, Saudi Arabia

\section{INTRODUCTION}

Endometrial cancer (EC) persists to be the most frequent malignancy of the female genital tract in developed countries, ${ }^{[1]}$ as well as in developing countries such as Saudi Arabia. ${ }^{[2]}$ Unfortunately, up to the present time, there are no standard tests available for early detection of EC. ${ }^{[3]}$

Address for correspondence: Prof. Dr. Ismail A. Al-Badawi, Section of Gynecologic Oncology, Department of Obstetrics and Gynecology, King Faisal Specialist Hospital and Research Centre, Riyadh 11211, Saudi Arabia.

E-mail: i_albadawi@yahoo.com
This is an open access journal, and articles are distributed under the terms of the Creative Commons Attribution-NonCommercial-ShareAlike 4.0 License, which allows others to remix, tweak, and build upon the work non-commercially, as long as appropriate credit is given and the new creations are licensed under the identical terms.

For reprints contact: reprints@ medknow.com

Cite this article as: Abu-Zaid A, Alsabban M, Alomar O, Abuzaid M, Jamjoom $\mathrm{MZ}$, Salem $\mathrm{H}$, et al. Preoperative cervical cytology as a prognostic factor in endometrioid-type endometrial cancer: A single-center experience from Saudi Arabia. Avicenna J Med 2020;10:111-17. 
Papanicolaou test, also known as pap smear and cervical cytology, has been efficiently used for decades for screening of cervical cancer. With high specificity ranging from $85 \%$ to $100 \%$, cervical cytology has permitted identification of premalignant and malignant lesions of the cervix. ${ }^{[4]}$ However, the utility of cervical cytology for the screening of EC is not a standardized practice worldwide..$^{[5]}$

Several studies have endeavored to address the use of cervical cytology in EC. ${ }^{[5-14]}$ Overall, these studies showed that cervical cytology could identify abnormal endometrial cells in patients with EC. ${ }^{[5-14]}$ Moreover, these studies revealed that abnormal endometrial cells in patients with EC correlated with poor survival and clinicopathological parameters, such as unfavorable histological tumor, high tumor grade, advanced stage disease, deep myometrial invasion (MI), and presence of lymphovascular space involvement (LVSI). From Saudi Arabia, to date, no similar study has been conducted.

Thus, our study has three main objectives: (1) to estimate the frequency of preoperative abnormal cervical cytology, (2) to explore correlations between preoperative cervical cytology and specific clinicopathological prognostic factors (tumor stage, endometrioid grade, MI depth, LVSI, cervical involvement, and recurrence), and (3) to examine the impact of preoperative cervical cytology on disease-free survival (DFS) and overall survival (OS) in Saudi patients with endometrioid-type EC.

\section{MATERIALS AND METHODS}

The study was conducted at the Department of Obstetrics and Gynecology, King Faisal Specialist Hospital and Research Centre, Riyadh, Saudi Arabia-a tertiary healthcare institution. The study protocol was approved by the institution's Research Advisory Council and Institutional Review Board.

The study's inclusion criteria included: (1) patients who underwent staging operation for EC from January 2010 to December 2014, (2) patients who had available cervical cytology results within 3 months before staging operation for $\mathrm{EC}$, and (3) patients with final histopathological diagnosis of endometrioid-type EC.

Afterward, the medical records of the study's research subjects were reviewed for a panel of clinical and pathological parameters, namely age, tumor staging according to the 2009 International Federation of Gynecology and Obstetrics (FIGO) staging system, ${ }^{[15]}$ the degree of tumor differentiation (histological grade), depth of MI, presence of LVSI, cervical involvement, recurrence, DFS, and OS.
In line with the institution's protocol, the cervical specimens were obtained with an endocervical cytobrush or cotton swab before staging operation. The cervical cytology results were reviewed by in-house pathologists and initially classified into normal, atypical (suspicious), or cancerous endometrial cells. Consistent with previous studies, ${ }^{[6,8,9]}$ the cervical cytology results were dichotomized into normal cytology (that is, normal endometrial cells) and abnormal cytology (including both atypical [suspicious] and cancerous [malignant] endometrial cells).

Numerical data were presented as mean \pm standard deviation (SD) (range: minimum-maximum). Categorical data were presented as number of cases $(n)$ and percentages (\%). Two-tailed Mann-Whitney $U$ test and chi-square tests were used for univariate analyses of continuous and categorical data, respectively. Survival analyses of DFS and OS were calculated according to the Kaplan-Meier estimates method and compared by using two-tailed log-rank test. Univariate and multivariate analyses of survival were performed using Cox proportional hazards model. All statistical analyses were performed using the Statistical Package for the Social Sciences (SPSS) software, version 22.0, for Windows (IBM, Armonk, New York). For all analyses, a $P$ value $<0.05$ was considered as statistically significant.

\section{RESULTS}

A total of 116 patients met the study's inclusion criteria. Table 1 depicts the patients' characteristics. The mean age \pm SD of patients was $58.5 \pm 10.3$ years (range: $36-90$ ). Endocervical cytobrush was the most frequently used sampling method ( $n=111,95.7 \%)$. Cervical cytology results were normal and abnormal in $70(60.3 \%)$ and 46 (39.7\%) patients, respectively. A greater proportion of patients had early FIGO stage I-II disease (91.4\%), low Grade I tumor (53.4\%), $\geq 50 \%$ depth of MI (71.6\%), negative LVSI (87.9\%), and negative cervical involvement (87.1\%). Among the patient cohort, tumor recurrence occurred in 11 patients (9.5\%), whereas death took place in six patients $(5.2 \%)$.

Table 2 depicts the univariate association between preoperative cervical cytology and some clinicopathological factors. Patients with abnormal cervical cytology had statistically significant higher rates of Grade II-III tumor $(P=0.004)$ and cervical involvement $(P=0.004)$ than patients with normal cervical cytology. There were no statistically significant differences identified between both groups with respect to FIGO stage, depth of MI, LVSI, tumor recurrence, and death $(P>0.05)$. 
Univariate Kaplan-Meier survival curves for mean DFS and OS rates, according to the preoperative cervical cytology results, are depicted in Figures 1 and 2, respectively. There were no statistically significant differences between patients with normal and abnormal cervical cytology results with regard to DFS (6.1 \pm 0.2 vs. $6.5 \pm 0.3$ years, respectively;

\begin{tabular}{|c|c|}
\hline Average age in years \pm standard deviation (range) & $58.5 \pm 10.3(36-99)$ \\
\hline \multicolumn{2}{|l|}{ Cervical cytology sampling method } \\
\hline Endocervical cytobrush, $n$ (\%) & III (95.7) \\
\hline Endocervical cotton swab, $n(\%)$ & $5(4.3)$ \\
\hline \multicolumn{2}{|l|}{ Cervical cytology results } \\
\hline Normal, $n(\%)$ & $70(60.3)$ \\
\hline Abnormal, $n(\%)$ & $46(39.7)$ \\
\hline \multicolumn{2}{|l|}{ FIGO stage } \\
\hline I-II, n (\%) & $106(91.4)$ \\
\hline III-IV, n (\%) & $10(8.6)$ \\
\hline \multicolumn{2}{|l|}{ Endometrioid grade } \\
\hline $\mathrm{I}, n(\%)$ & $62(53.4)$ \\
\hline II-III, $n(\%)$ & $54(46.6)$ \\
\hline \multicolumn{2}{|l|}{ Myometrial invasion depth } \\
\hline$<50 \%, n(\%)$ & $83(71.6)$ \\
\hline$\geq 50 \%, n(\%)$ & $33(28.4)$ \\
\hline \multicolumn{2}{|l|}{ Lymphovascular space invasion } \\
\hline No, $n(\%)$ & $102(87.9)$ \\
\hline Yes, $n(\%)$ & $14(12.1)$ \\
\hline \multicolumn{2}{|l|}{ Cervical involvement } \\
\hline No, $n(\%)$ & I0I (87.I) \\
\hline Yes, $n(\%)$ & $15(12.9)$ \\
\hline \multicolumn{2}{|l|}{ Tumor recurrence } \\
\hline No, $n(\%)$ & $105(90.5)$ \\
\hline Yes, $n(\%)$ & II (9.5) \\
\hline \multicolumn{2}{|l|}{ Patient death } \\
\hline No, $n(\%)$ & 110 (94.8) \\
\hline Yes, $n(\%)$ & $6(5.2)$ \\
\hline
\end{tabular}

$P=0.525)$ and $O S(0.6 \pm 0.01$ vs. $2.2 \pm 0.1$ months, respectively; $P=0.166$ ). Furthermore, multivariate analyses of DFS and OS failed to show preoperative cervical cytology as a significant independent prognostic factor of DFS and OS $(P>0.05$, data not shown).

\section{DISCUSSION}

The use of cervical cytology, in developed countries, has been shown to substantially reduce the incidence and mortality rates of cervical cancer by approximately $70 \%-80 \% \cdot{ }^{[16,17]}$ There is an anatomical connection between the uterine cavity and the cervix. Thus, the evaluation of biological materials shed from the upper (uterine cavity) to the lower (cervix) genital tract using cervical cytology presents a plausible opportunity to potentially identify uterine lesions. ${ }^{[3]}$ Several studies have scrutinized the value of preoperative cervical cytology in EC in terms of screening, prognostic marker of clinicopathological outcomes, and survival. ${ }^{[5-14]}$

A recent systematic review and meta-analysis comprising 45 studies and nearly 6600 patients with EC showed that abnormal cervical cytology results were identified in $45 \%$ of patients with EC before diagnosis or surgery. ${ }^{[18]}$ The absence of adequate yield of endocervical cells renders the cervical cytology unsatisfactory. Accumulating evidence from several studies showed that cervical sampling with endocervical cytobrush was more superior to cotton swab in obtaining adequate yield of endocervical cells. ${ }^{[19-23]}$ Harrison et al. ${ }^{[24]}$ concluded that endocervical brushes were cost-

\begin{tabular}{|c|c|c|c|}
\hline \multirow[t]{2}{*}{ Variable } & \multicolumn{2}{|c|}{ Cervical cytology result } & \multirow[t]{2}{*}{ Univariate, $P$ value $f$} \\
\hline & Normal, $n=70$ & Abnormal, $n=46$ & \\
\hline FIGO stage & & & 0.169 \\
\hline $\mathrm{I}-\mathrm{II}, n(\%)$ & $66(62.3)$ & $40(37.7)$ & \\
\hline III-IV, n (\%) & $4(40)$ & $6(60)$ & \\
\hline Endometrioid grade & & & 0.004 \\
\hline $\mathrm{I}, n(\%)$ & $45(72.6)$ & I7 (27.4) & \\
\hline II-III, $n(\%)$ & $25(46.3)$ & $29(53.7)$ & \\
\hline Myometrial invasion depth & & & 0.099 \\
\hline$<50 \%, n(\%)$ & $54(65.1)$ & $29(39.9)$ & \\
\hline$\geq 50 \%, n(\%)$ & $16(48.5)$ & $17(5 \mathrm{I} .5)$ & \\
\hline Lymphovascular space invasion & & & 0.794 \\
\hline No, $n(\%)$ & $62(60.8)$ & $40(39.2)$ & \\
\hline Yes, $n(\%)$ & $8(57 I)$ & $6(42.9)$ & \\
\hline Cervical involvement & & & 0.004 \\
\hline No, $n(\%)$ & $66(65.3)$ & $35(34.7)$ & \\
\hline Yes, $n(\%)$ & $4(26.7)$ & II (73.3) & \\
\hline Tumor recurrence & & & 0.815 \\
\hline No, $n(\%)$ & $63(60)$ & $42(40)$ & \\
\hline Yes, $n(\%)$ & $7(63.6)$ & $4(36.4)$ & \\
\hline Patient death & & & 0.165 \\
\hline No, $n(\%)$ & $68(6 I .8)$ & $42(38.2)$ & \\
\hline Yes, $n(\%)$ & $2(33.3)$ & $4(66.7)$ & \\
\hline
\end{tabular}


effective than cotton swabs by decreasing the requirement to repeat cervical sampling due to the lack of adequate yield of endocervical cells. In our study, the majority of patients (95.7\%) had the endocervical cytobrush as the most frequently used sampling method for cervical cytology. Moreover, the frequency of abnormal cervical cytology (suspicious and malignant cells) was $39.7 \%$. This proportion was consistent with the published literature ranging from as low as $30 \%$ to as high as $70 \% .^{[5-14]}$

Cervical cytology can prove to be technically difficult in discerning malignant and benign lesions of the endometrium. ${ }^{[5]}$ Moreover, neoplastic cells usually do not exfoliate readily from the endometrium, and this may contribute to poor detection rates of abnormal cervical cytology ${ }^{[5]}$ Nevertheless, it has been shown that the severity

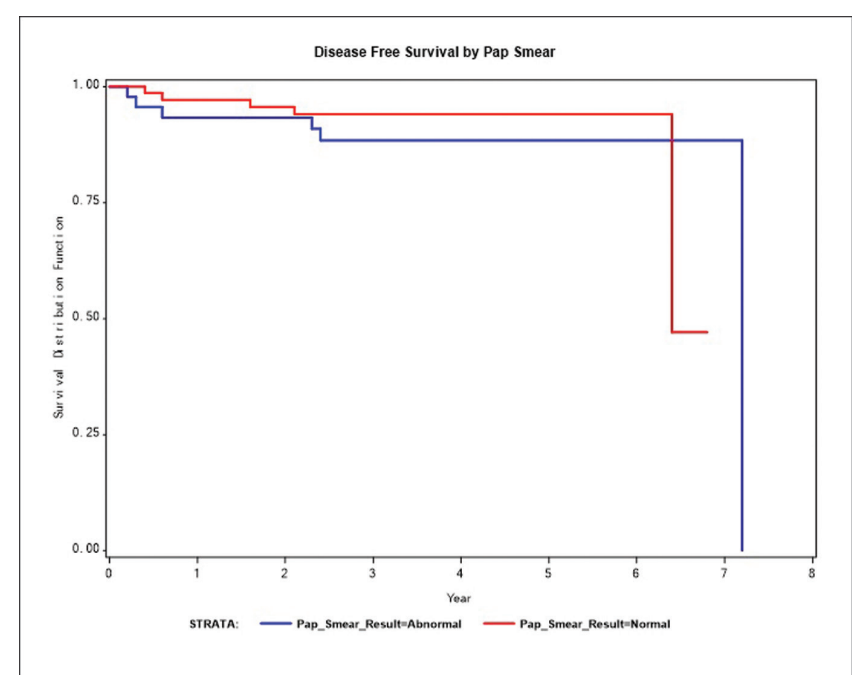

Figure 1: Disease-free survival according to preoperative cervical cytology in patients with endometrioid-type endometrial cancer $(n=116)$.

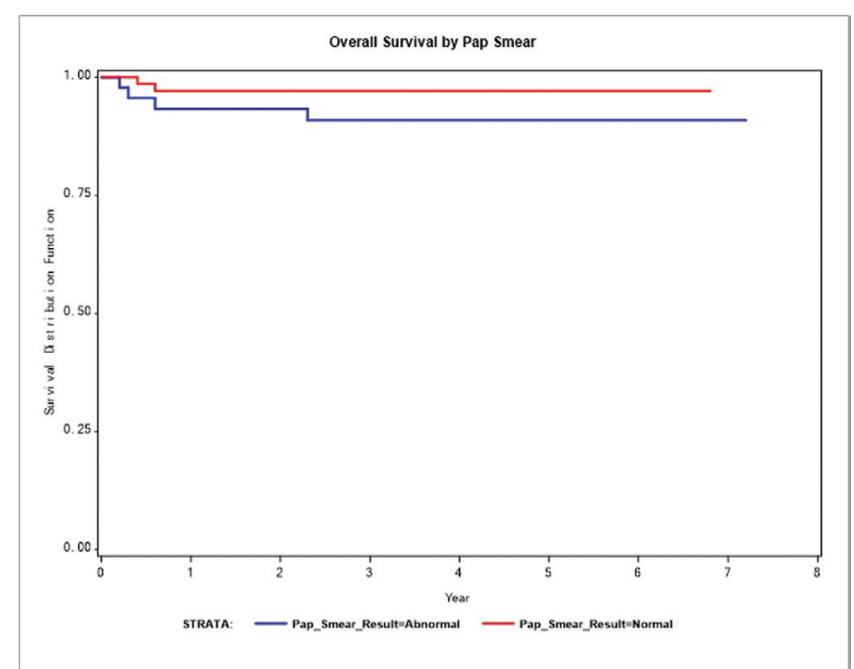

Figure 2: Overall survival according to preoperative cervical cytology in patients with endometrioid-type endometrial cancer $(n=116)$. of EC correlates positively with higher detection rates of abnormal endocervical cells. ${ }^{[18,23]}$ In particular, high-grade EC lesions are highly likely to result in greater detection rates of abnormal endocervical cells due to their large sizes, hyperchromatic nuclei, prominent nucleoli, and background tumor diathesis; all of which trigger suspicions to look for atypical cells in the cervical cytology. ${ }^{[5]}$ In addition, the presence of increased histiocytes in cervical cytology correlates positively with higher detection rates of abnormal endocervical cells as well as significant endometrial lesions, such as sarcomas, carcinomas, and polyps. ${ }^{[25]}$

The cervical cytology findings of atypical endometrial glandular cells in a 59-year-old woman who had a postoperative histology of Grade I endometrioid-type EC

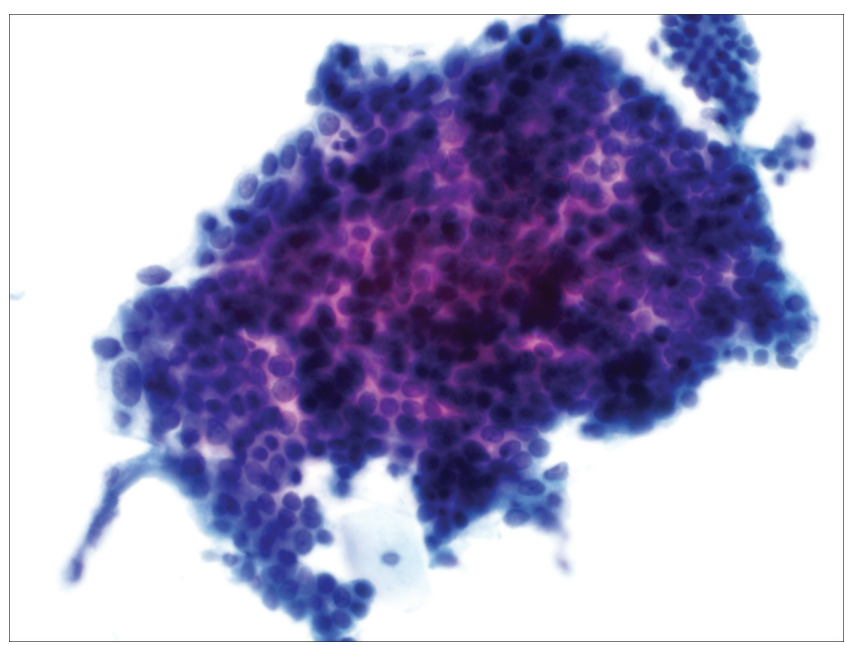

Figure 3: Cervical cytology showing atypical endometrial glandular cells in a 59-year-old woman. The cells were arranged in clusters and showed mild nuclear polymorphism with coarse granular chromatin, macronucleoli and increased nuclear-to-cytoplasmic ratio.

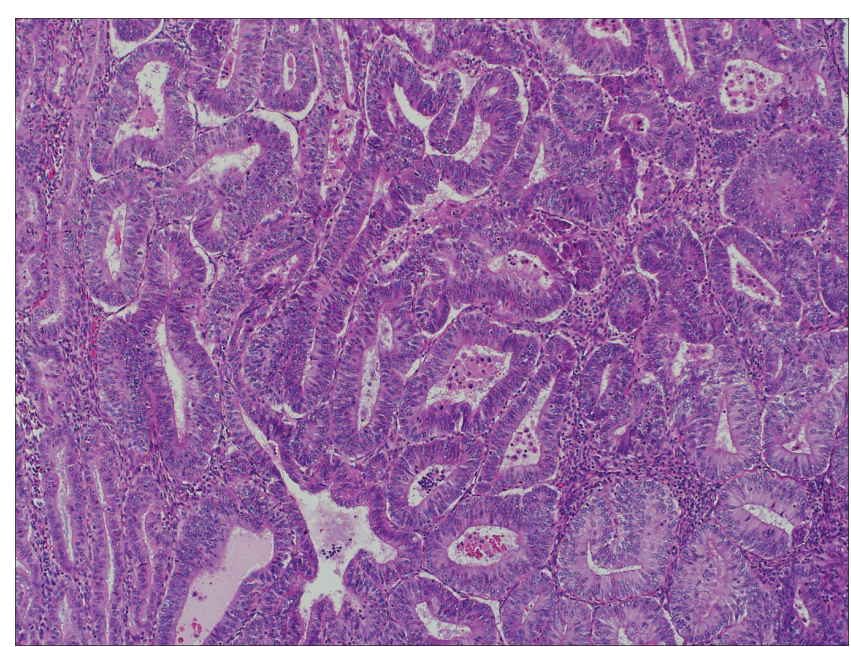

Figure 4: Histopathology showing endometrioid-type endometrial adenocarcinoma in a 59-year-old woman. The irregular endometrial-type glands were lined by columnar epithelium with mild cytologic atypia. The tumor contained less than $5 \%$ of the overall solid areas and was classified as FIGO Grade I tumor. FIGO = International Federation of Gynecology and Obstetrics. 
are depicted in Figures 3 and 4, respectively. The cervical cytology findings of malignant endometrial cells in a 45-year-old woman who had a postoperative histology of Grade II endometrioid-type EC are depicted in Figures 5 and 6 , respectively.

Previous reports showed that preoperative cervical cytology significantly correlated with numerous clinicopathological parameters in patients with EC [Table 3]. ${ }^{[5-11,14]}$ Examples of such parameters included tumor histology, tumor grade, tumor stage, depth of MI, lymph node involvement, cervical involvement, peritoneal washing, and LVSI. The findings of our study matched the findings of earlier reports only with regard to tumor grade and cervical involvement. There are three potential reasons that may account for this observation between our study and others. First, our study included a small sample size $(n=116)$, which may have negatively influenced the results by decreasing the power of study. Second, our study included only patients with endometrioid-type EC histology, which is naturally known to harbor a better prognosis than non-endometrioid-type EC histology. Third, the majority of patients had initially "favorable" EC, in terms of early stage disease (91.4\%), reduced $<50 \%$ depth of MI $(71.6 \%)$, and absence of LVSI (87.1\%); this patient selection may have skewed the results and masked the power of study to identify significant correlations.

In our study, we did not examine the impact of bulky tumor volumes versus small focal ones on the yield of abnormal cervical cytology. Two previous studies showed that larger tumor size $(\geq 3 \mathrm{~cm})$ was positively associated with higher rates of abnormal cervical cytology in patients with EC. ${ }^{[11,26]}$ However, this finding was not echoed in a retrospective Korean study of 108 patients, which reported no statistically

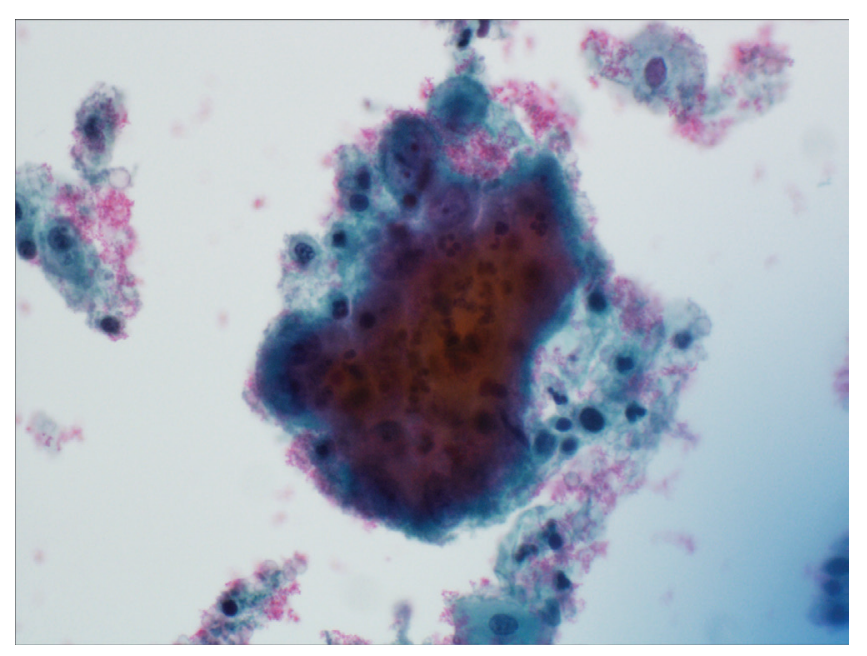

Figure 5: Cervical cytology showing malignant endometrial cells in a 45-yearold woman. The cells were arranged in nests and had increased nuclear-tocytoplasmic ratio with moderate nuclear pleomorphism, multinucleation, and tumor diathesis in the background. significant association between preoperative cervical cytology and postoperative tumor size. ${ }^{[14]}$

Abnormal cervical cytology has been shown to correlate with an increased risk of recurrence in patients with nonendometrioid-type EC, such as clear cell carcinoma and papillary serous carcinoma. ${ }^{[12,13]}$ Conversely, in patients with endometrioid-type EC, the risk of recurrence does not seem to be increased in patients with abnormal cervical cytology. ${ }^{[13]}$ The latter finding was reciprocated in our study in univariate and multivariate analyses. It appears that tumor histology plays a key role in modulating the hazard of recurrence in EC patients with preoperative abnormal cervical cytology.

Some reports suggested that cervical cytology can influence the decision of extended surgical staging (or debulking) in patients with EC. To elaborate, DuBeshter et al. ${ }^{[6]}$ claimed that pelvic and para-aortic lymphadenectomy could be omitted in patients with normal cervical cytology and Grade I EC. On the contrary, Larson et al..$^{[7]}$ stated that preoperative abnormal cervical cytology is associated with an increased risk of aggressive disease with lymph node spread, and thus advised for indispensable lymphadenectomy in such cases. Whether preoperative cervical cytology can substantially impact management decisions continues to be a point of controversy. ${ }^{[8]}$

Not much is known about the impact of preoperative cervical cytology on survival. Our study failed to show abnormal cervical cytology as a significant independent prognostic factor of DFS in univariate analysis. Our finding was mirrored in two studies, ${ }^{[8,14]}$ and differed in another study that examined only high-risk and high-grade patients with EC. ${ }^{[12]}$

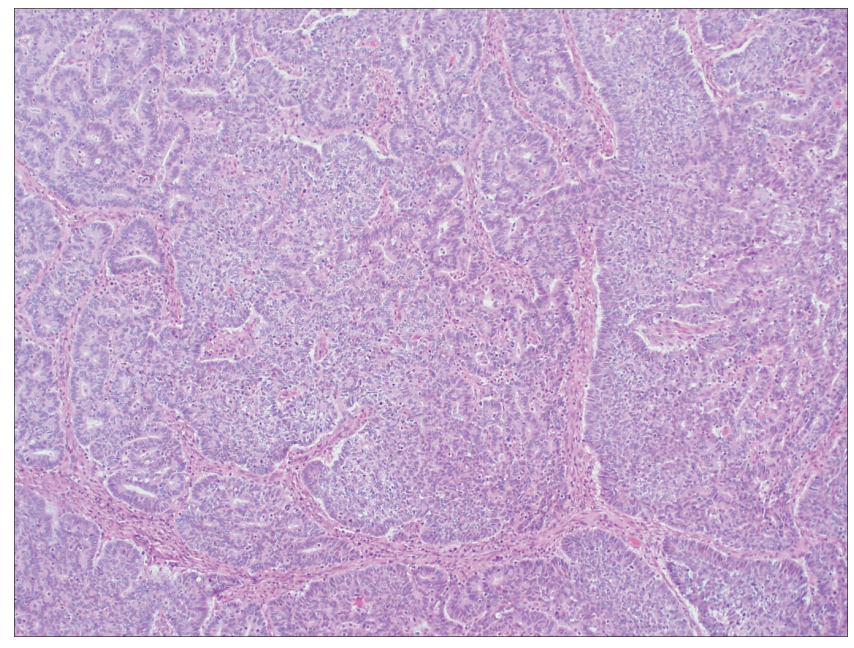

Figure 6: Histopathology showing endometrioid-type endometrial adenocarcinoma in a 45-year-old woman. The neoplastic tumor cells were arranged in a sheet-like manner without glandular features. There were moderate nuclear pleomorphisms with prominent nucleoli. The tumor contained around $40 \%$ of the overall solid areas and was classified as FIGO Grade II tumor. FIGO = International Federation of Gynecology and Obstetrics. 


\begin{tabular}{|c|c|c|c|c|c|c|c|}
\hline Ref. & Authors & Year & Stage & Grade & Histology & Depth of MI & LN involvement \\
\hline$[6]$ & DuBeshter et al. & 1991 & Yes & Yes & NE & Yes & Yes \\
\hline$[7]$ & Larson et al. & 1994 & Yes & Yes & Yes & $\mathrm{NE}$ & Yes \\
\hline [8] & Fukuda et al. & 1999 & Yes & Yes & Yes & Yes & Yes \\
\hline [9] & Gu et al. & 2001 & Yes & Yes & $\mathrm{NE}$ & No & $\mathrm{NE}$ \\
\hline$[14]$ & Shin et al. & 2009 & Yes & Yes & Yes & Yes & No \\
\hline$[10]$ & Lai et al. & 2015 & Yes & Yes & Yes & $\mathrm{NE}$ & NE \\
\hline$[11]$ & Serdy et al. & 2016 & Yes & NE & Yes & Yes & Yes \\
\hline \multirow[t]{2}{*}{ [5] } & Nadaf et al. & 2017 & No & Yes & No & No & NE \\
\hline & Present study & 2020 & No & Yes & NE & No & NE \\
\hline
\end{tabular}

$\mathrm{LN}=$ lymph node, $\mathrm{MI}=$ myometrial invasion, $\mathrm{NE}=$ not evaluated, Ref = reference

"Yes" and "No" refers to statistically significant chi-square correlations between cervical cytology and the specific clinicopathological parameter in the referenced study. A $P$ value $<0.05$ was determined as statistically significant

In Saudi Arabia, the utility of preoperative cervical cytology in patients with EC is not a standard protocol. However, we reported the first ever study of such experience from Saudi Arabia, and more single-center experiences are encouraged to report the same. Strengths of our study include the uniform endometrioid-type histology and survival analyses of DFS and OS. All in all, our study enriches the existing literature with additional findings from an intrinsically different cohort of patients with EC. Nonetheless, our study is not without limitations. Such limitations include the relatively small sample size and lack of evaluation of other important prognostic parameters, for example, lymph node spread.

\section{CONCLUSION}

In our study, the frequency of abnormal cervical cytology in patients with endometrioid-type EC is not uncommon. Moreover, abnormal cervical cytology correlates with poor prognostic factors, namely high tumor grade and cervical involvement. However, cervical cytology does not seem to be a significant independent prognostic factor of survival. Despite the cervical cytology's potential prediction of poor prognostic factors in patients with EC, its use as a preoperative workup tool is controversial in its value.

\section{Financial support and sponsorship}

Nil.

\section{Conflicts of interest}

There are no conflicts of interest.

\section{REFERENCES}

1. Siegel RL, Miller KD, Jemal A. Cancer statistics, 2019. CA Cancer J Clin 2019;69:7-34.

2. Althubiti MA, Nour Eldein MM. Trends in the incidence and mortality of cancer in Saudi Arabia. Saudi Med J 2018;39:1259-62.

3. Costas L, Frias-Gomez J, Guardiola M, Benavente Y, Pineda M, Pavón MÁ, et al.; Screenwide Team. New perspectives on screening and early detection of endometrial cancer. Int J Cancer 2019;145:3194-206.
4. Nanda K, McCrory DC, Myers ER, Bastian LA, Hasselblad V, Hickey JD, et al. Accuracy of the Papanicolaou test in screening for and follow-up of cervical cytologic abnormalities: A systematic review. Ann Intern Med 2000;132:810-9.

5. Nadaf A, Rani H, S S P, Rao R, Shastri D. Pap smears in endometrial adenocarcinoma: Does it have a role? Asian Pac J Cancer Prev 2017; 18:1145-50.

6. DuBeshter B, Warshal DP, Angel C, Dvoretsky PM, Lin JY, Raubertas RF. Endometrial carcinoma: The relevance of cervical cytology. Obstet Gynecol 1991;77:458-62.

7. Larson DM, Johnson KK, Reyes CN Jr, Broste SK. Prognostic significance of malignant cervical cytology in patients with endometrial cancer. Obstet Gynecol 1994;84:399-403.

8. Fukuda K, Mori M, Uchiyama M, Iwai K, Iwasaka T, Sugimori H, et al. Preoperative cervical cytology in endometrial carcinoma and its clinicopathologic relevance. Gynecol Oncol 1999;72: 273-7.

9. Gu M, Shi W, Barakat RR, Thaler HT, Saigo PE. Pap smears in women with endometrial carcinoma. Acta Cytol 2001;45:555-60.

10. Lai CR, Hsu CY, Hang JF, Li AF. The diagnostic value of routine Papanicolaou smears for detecting endometrial cancers: An update. Acta Cytol 2015;59:315-8.

11. Serdy K, Yildiz-Aktas I, Li Z, Zhao C. The value of Papanicolaou tests in the diagnosis of endometrial carcinoma: A large study cohort from an academic medical center. Am J Clin Pathol 2016;145: 350-4.

12. Amkreutz LCM, Pijnenborg JMA, Joosten DWL, Mertens HJMM, Van Kuijk SMJ, Engelen MJA, et al. Contribution of cervical cytology in the diagnostic work-up of patients with endometrial cancer. Cytopathology 2018;29:63-70.

13. Brown AK, Gillis S, Deuel C, Angel C, Glantz C, Dubeshter B. Abnormal cervical cytology: A risk factor for endometrial cancer recurrence. Int J Gynecol Cancer 2005;15:517-22.

14. Shin DH, Choi KU, Suh DS, Yoon MS, Kim JY. Implications of preoperative cervical cytology in endometrial carcinoma. Basic Applied Path 2009;2:30-4.

15. Pecorelli S. Revised FIGO staging for carcinoma of the vulva, cervix, and endometrium. Int J Gynaecol Obstet 2009;105:103-4.

16. Nieminen P, Kallio M, Hakama M. The effect of mass screening on incidence and mortality of squamous and adenocarcinoma of cervix uteri. Obstet Gynecol 1995;85:1017-21.

17. Kitchener HC, Symonds P. Detection of cervical intraepithelial neoplasia in developing countries. Lancet 1999;353:856-7.

18. Frias-Gomez J, Benavente $\mathrm{Y}$, Ponce J, Brunet J, Ibanez R, Peremiquel-Trillas $\mathrm{P}$, et al. Sensitivity of cervico-vaginal cytology in endometrial carcinoma: A systematic review and meta-analysis. Cancer Cytopathol2020. doi: 10.1002/cncy.22266. [Epub ahead of print]. 
19. Koonings PP, Dickinson K, d'Ablaing G $3^{\text {rd }}$, Schlaerth JB. A randomized clinical trial comparing the cytobrush and cotton swab for Papanicolaou smears. Obstet Gynecol 1992;80:241-5.

20. Pretorius RG, Sadeghi M, Fotheringham N, Semrad N, Watring WG. A randomized trial of three methods of obtaining Papanicolaou smears. Obstet Gynecol 1991;78:831-6.

21. Chalvardjian A, De Marchi WG, Bell V, Nishikawa R. Improved endocervical sampling with the cytobrush. CMAJ 1991;144: 313-7.

22. Kavak ZN, Eren F, Pekin S, Külliu S. A randomized comparison of the 3 Papanicolaou smear collection methods. Aust N Z J Obstet Gynaecol 1995;35:446-9.
23. Martin-Hirsch P, Jarvis G, Kitchener H, Lilford R. Collection devices for obtaining cervical cytology samples. Cochrane Database Syst Rev 2000:Cd001036.

24. Harrison DD, Hernandez E, Dunton CJ. Endocervical brush versus cotton swab for obtaining cervical smears at a clinic. A cost comparison. J Reprod Med 1993;38:285-8.

25. Nassar A, Fleisher SR, Nasuti JF. Value of histiocyte detection in pap smears for predicting endometrial pathology. An institutional experience. Acta Cytol 2003;47:762-7.

26. Zhou J, TomashefskiJF Jr, Khiyami A. Thinprep pap tests in patients with endometrial cancer: A histo-cytological correlation. Diagn Cytopathol 2007;35:448-53. 